\title{
Validar: a testbed for advanced 2-micron Doppler lidar
}

\author{
Grady J. Koch*a, Mulugeta Petros ${ }^{\mathrm{b}}$, Bruce W. Barnes ${ }^{\mathrm{a}}$, Jeffrey Y. Beyon ${ }^{\mathrm{c}}$, Farzin Amzajerdian ${ }^{\mathrm{a}}$, \\ Jirong $\mathrm{Yu}^{\mathrm{a}}$, Michael J. Kavaya ${ }^{\mathrm{a}}$, and Upendra N. Singh ${ }^{\mathrm{a}}$ \\ ${ }^{a}$ NASA Langley Research Center, MS 468, Hampton, VA USA 23681 \\ ${ }^{\mathrm{b}}$ Science and Technology Corporation, 10 Basil Sawyer Drive, Hampton, VA USA 23666 \\ ${ }^{c}$ California State University., Dept. of Electrical and Computer Engineering, Los Angeles, CA USA
}

\begin{abstract}
High-energy 2- $\mu \mathrm{m}$ lasers have been incorporated in a breadboard coherent Doppler lidar to test component technologies and explore applications for remote sensing of the atmosphere. Design of the lidar is presented including aspects in the laser transmitter, receiver, photodetector, and signal processing. Sample data is presented on wind profiling and $\mathrm{CO}_{2}$ concentration measurements.
\end{abstract}

Keywords: Lidar, coherent lidar, DIAL, wind, solid-state lasers

\section{INTRODUCTION}

Recent advances in high-energy $2-\mu \mathrm{m}$ solid-state laser technology have enabled lidar applications for long range wind sensing and $\mathrm{CO}_{2}$ measurement. Wind profiling with $2-\mu \mathrm{m}$ lidar has already shown merit using low energy laser transmitters, and the addition of stronger energy lasers promises to boost the applicability of the coherent Doppler technique to farther distances and higher altitudes. ${ }^{1,2,3}$ Research at NASA is aimed at developing lidar technology for satellite-based observation of wind on a global scale. The ability to profile tropospheric wind is a key measurement for understanding and predicting atmospheric dynamics and is a critical measurement for improving weather forecasting and climate model validation. ${ }^{4}$ Homeland security applications have been proposed to provide wind data for accurate transport prediction of a plume release of a nuclear, biological, or chemical agent. ${ }^{5}$ Safe and effective military operations are also dependent on weather forecasting and wind profiling.

Another application for 2- $\mu \mathrm{m}$ lidar is remote sensing of $\mathrm{CO}_{2}$ by the differential absorption lidar (DIAL) technique. Atmospheric $\mathrm{CO}_{2}$ concentration is of interest to climate change and global warming studies. The recent rate of increase of $\mathrm{CO}_{2}$ is about 100 times higher than at any other time during the last 100 years. While the long-term trend of $\mathrm{CO}_{2}$ in the atmosphere and its seasonal variations are well documented, the sources and sinks of $\mathrm{CO}_{2}$ on continental and regional scales are not well understood. ${ }^{6}$ A sink for $\mathrm{CO}_{2}$ comprising more than $25 \%$ of the 7.1 petagrams $\left(10^{17}\right.$ grams) of $\mathrm{CO}_{2}$ that is released annually into the atmosphere, remains unaccounted for. ${ }^{7}$ It is also known that both land and ocean sinks fluctuate with space and time. Knowledge of the spatial and temporal distributions of $\mathrm{CO}_{2}$ and an understanding of the causes of these variations are essential in predicting future levels of $\mathrm{CO}_{2}$ and their impact of climate change. Differential absorption lidar (DIAL) potentially offers several advantages over passive techniques for remote $\mathrm{CO}_{2}$ sensing. These advantages include relatively high spatial resolution, high measurement specificity (avoiding interference from other gases), lack of dependence on external light sources, and relatively simple inversion methods compared to retrieval methods used in passive remote sensing. A challenge to lidar in this application is that the concentration measurement must be made with a very high level of precision and accuracy. To assist the understanding of many carbon cycle processes a $\mathrm{CO}_{2}$ measurement accuracy of 1-2 ppm over 1-2 km vertical range intervals in the troposphere and column measurements with $1-2 \mathrm{ppm}$ precision are ultimately needed. 
We report here on technology developments and lidar system demonstrations aimed at both wind and $\mathrm{CO}_{2}$ applications. High-energy Ho:Tm:YLF and Ho:Tm:LuLiF laser transmitters are described along with aspects of injection seeding, wavelength tuning, and wavelength stabilization. Coherent heterodyne receivers are also discussed, including advances with an integrated photodiode/preamp in a dual-balanced configuration. A complete lidar system testbed has been built from these component and integrated with a real-time signal processing and display computer. Sample wind and $\mathrm{CO}_{2}$ measurements will be shown. Validar gets its name from the concept of "validation lidar," in that it can serve as a calibration and validation source for future airborne and spaceborne lidar missions. Validar is housed within a mobile trailer for field measurements.

\section{LIDAR DESIGN}

The optical layout of Validar is shown in Figure 1 and a list of specifications is given in Table 1. Transmitter and receiver subsystems are each described separately, with the transmitter considered to consist of the pulsed laser, continuous wave $(\mathrm{CW})$ laser, and the optics associated with coupling the two. The receiver is considered to be the transmit/receive switch, telescope, and photodetector. Not shown in Figure 1 is a scanner mounted through the roof of the trailer for steering the lidar beam.

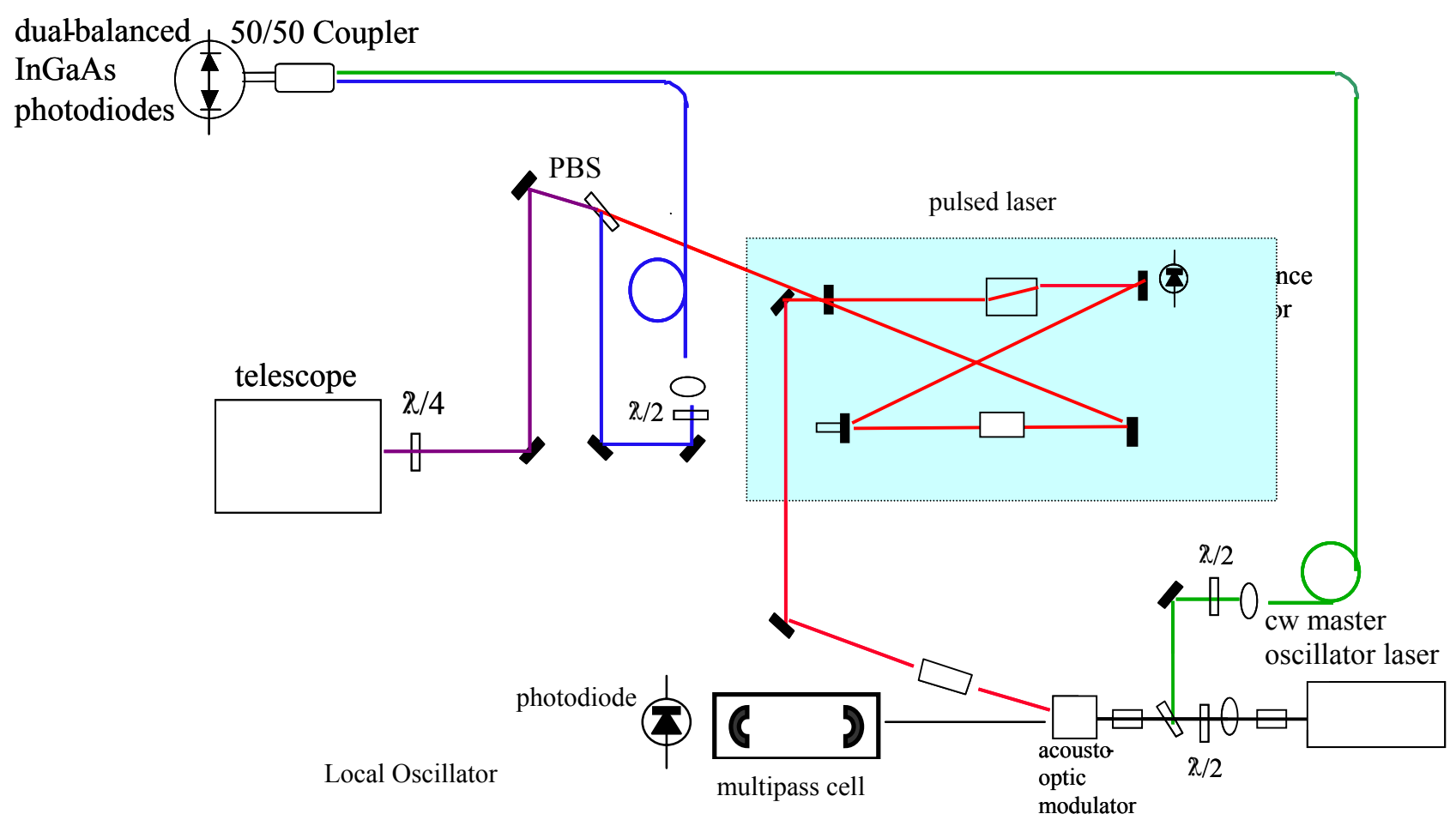

Figure 1: Optical layout of the lidar.

\subsection{Laser Transmitter}

The 2- $\mu \mathrm{m}$ wavelength is advantageous in a coherent Doppler lidar for several reasons. First, it offers a high level of eyesafety with a maximum permissible exposure of $100 \mathrm{~mJ} / \mathrm{cm}^{2} .8$ Second, the $2-\mu \mathrm{m}$ wavelength is strongly 
backscattered by aerosols in the atmosphere, from which the Doppler shift indicates wind speed. Third, holmium solidstate lasers can produce a high level of pulse energy at $2.05 \mu \mathrm{m}$. Last, $\mathrm{CO}_{2}$ has absorption lines at this wavelength that are well suited to DIAL in absorption line strength, insensitivity to temperature, and freedom from interference by other gases.

A holmium laser material, with an activator of thulium, is used to generate the $2-\mu \mathrm{m}$ wavelength. The host material of yttrium lithium fluoride (YLF) has proven robust under strong pumping conditions with a low level of thermal lensing. Tests have shown as much as $600 \mathrm{~mJ}$ with a single frequency spectrum using Ho:Tm:YLF. ${ }^{9}$ However, recent research has proven that the host material of lutetium lithium fluoride (LuLiF) can produce more energy that YLF for the same pumping configuration. ${ }^{10}$ Validar is now being converted to operation with Ho:Tm:LuLiF, but the atmospheric measurements shown in Section 3 were taken using Ho:Tm:YLF. The new configuration with Ho:Tm:LuLiF should extend the range capability of the instrument with the availability of higher pulse energy.

\begin{tabular}{|lll|}
\hline & Reported Configuration* & Upgraded Configuration \\
& & \\
Laser material: & $\mathrm{Ho}: \mathrm{Tm}: \mathrm{YLF}$ & $\mathrm{Ho}: \mathrm{Tm}: \mathrm{LuLiF}$ \\
Pulse energy: & $75 \mathrm{~mJ}$ & $95 \mathrm{~mJ}$ \\
Pulse width: & $150 \mathrm{~ns}$ & $140 \mathrm{~ns}$ \\
Pulse repetition rate: & $5-10 \mathrm{~Hz}$ & $5-10 \mathrm{~Hz}$ \\
Spectrum: & single frequency & single frequency \\
Wavelength: & $2051.0 \mathrm{~nm}$ & $2053.5 \mathrm{~nm}$ \\
& & $3-4 \mathrm{~nm}$ including $\mathrm{CO}_{2}$ lines \\
Wavelength tuning: & $3-4 \mathrm{~nm}$ including $\mathrm{CO}_{2}$ lines & $<1.3$ times diffraction limit \\
Beam quality: & $<1.3$ times diffraction limit & \\
Long term (one hour) & & not yet tested \\
wavelength stability: & $<16.5 \mathrm{MHz}$ (for $\mathrm{CO}_{2}$ DIAL application) \\
Wavelength accuracy: & $<15 \mathrm{MHz}$ (for $\mathrm{CO}_{2}$ DIAL application) & not yet tested \\
Detector & InGaAs in heterodyne configuration & InGaAs in heterodyne config. \\
Telescope aperture: & 4 inches & 6 inches \\
Scanner: & 8.5 inch aperture, & 8.5 inch aperture, \\
& full hemispherical coverage & full hemispherical coverage \\
& & \\
Table 1: Specifications of Validar. & $*$ Data in Section 3 was taken with the Reported Configuration. \\
\hline
\end{tabular}

The Ho:Tm:LuLiF rod is contained in a pump head using conductively cooled laser diode arrays. Water cooling is used for the heat sink of the diode arrays, but the laser diodes are not directly cooled with water. Six AlGaAs diode arrays side pump the laser rod; each array has six bars that can provide up to $600 \mathrm{~mJ}$ near $792 \mathrm{~nm}$ in a 1-ms pulse. Three sets of two diode arrays, side by side, are arranged $120^{\circ}$ apart around the laser rod circumference. The laser rod has a diameter of $4 \mathrm{~mm}$ and is encased in a 6-mm outer-diameter fused-silica glass tube for water cooling of the laser rod; the coolant temperature is kept at $15 \mathrm{C}$. The $20-\mathrm{mm}$ long laser rod is doped with $6 \% \mathrm{Tm}$ and $0.4 \% \mathrm{Ho}$. The resonator is in a ring configuration with a total length of $2.0 \mathrm{~m}$, with an acousto-optic Q-switch to spoil the laser cavity.

A continuous wave (CW) Ho:Tm:YLF laser serves as both an injection seed source and a local oscillator. This laser was originally built by Coherent Technologies, Inc. for the Space Readiness Coherent Lidar Experiment (SPARCLE). ${ }^{11}$ Approximately $10 \mathrm{~mW}$ of this $\mathrm{CW}$ laser is split off and focused into a polarization maintaining optical fiber for the local oscillator channel. The rest of the CW energy is used for injection seeding after being frequency shifted $105 \mathrm{MHz}$ by an acousto-optic modulator. Such an offset sets an intermediate frequency between the local oscillator and pulsed laser output. A series of three Faraday isolators are used between the $\mathrm{CW}$ laser and the pulsed laser in order to prevent damage to the $\mathrm{CW}$ laser from a counter-propagating laser pulse and to isolate the local oscillator channel from influences by the laser pulse and acousto-optic modulator. 
To ensure a single-frequency pulsed output by the injection seeding process, the pulse laser cavity is actively matched to the frequency of the injection seed by a ramp-and-fire technique. ${ }^{12}$ A piezo-electric translator (PZT) mounted to a mirror of the pulsed laser is ramped by voltage, as shown in Figure 2. An InGaAs photodiode circuit views the resonance of the injection seed with the pulsed laser cavity, and resonance peaks occur when the pulsed laser cavity length matches the injection seed frequency. A circuit senses the resonance and fires the Q-switch when this cavity matching condition is determined. This results is a single-frequency output spectrum from the pulsed laser. Successful seeding can be determined by a smooth shape to the laser pulsed, as shown in Figure 2, and a doubling of output power as the ring resonator is forced to run in a single direction.

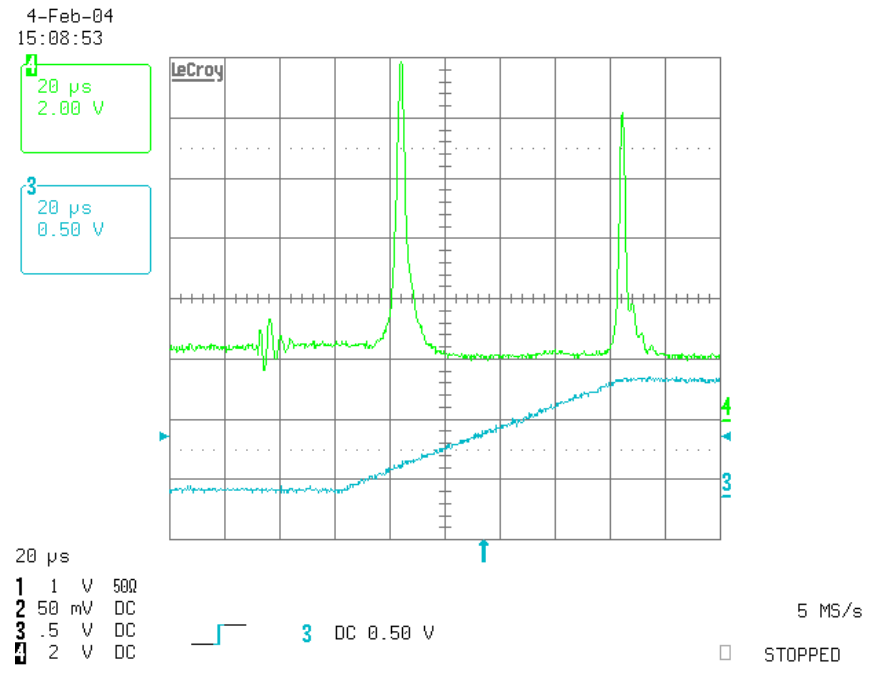

Figure 2: Signals involved in resonance matching. The lower trace is a ramp voltage applied to a PZT on a pulsed laser cavity mirror. Resonance occurs (upper trace) when the seed laser matches a mode of the pulsed laser.

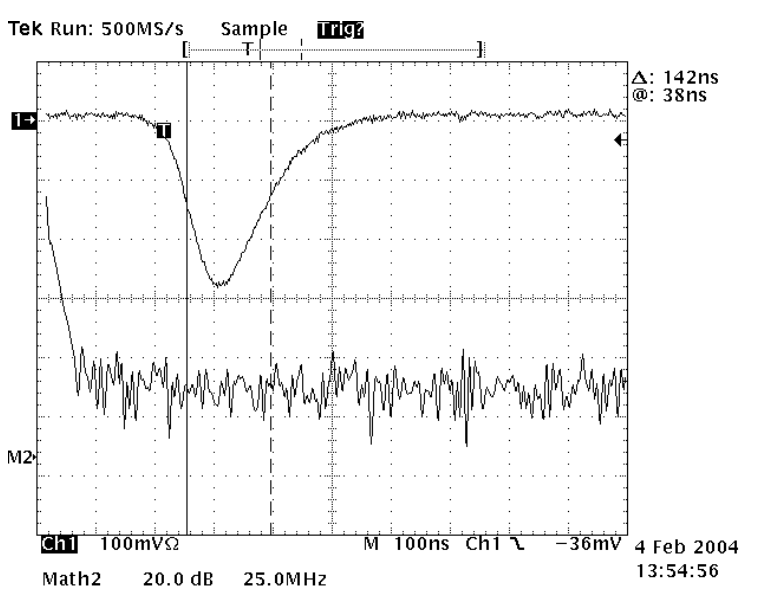

Figure 3: Single-frequency output pulse viewed in time domain (upper trace) and in frequency domain (lower trace). No mode beating is observed.

Control of the wavelength of the $\mathrm{CW}$ laser has been shown to subsequently control the pulsed laser. For $\mathrm{CO}_{2}$ measurement applications, knowledge and control of the wavelength is critical. DIAL uses two wavelengths: one matched to an absorption line of $\mathrm{CO}_{2}$ and one tuned off of the absorption peak. A technique has been developed for stabilizing CW 2- $\mu \mathrm{m}$ lasers to absorption peaks by a wavelength modulation spectroscopic technique. ${ }^{13}$ Modulation of the laser wavelength, and tuning control for feedback stabilization, is provided by a PZT mounted to the output coupler of the CW laser. Experiments showed that the laser's frequency can be held to within a few percent of the absorption linewidth. Further research verified that the pulsed laser is closely locked to the seed laser wavelength so that the pulsed laser wavelength is stabilized to the absorption line as the seed laser is stabilized. ${ }^{14}$ DIAL measurements also require that the wavelength on and off the absorption line be alternated. In the data of Section 3 and in a more complete report in preparation, we alternate wavelength at the pulse repetition rate of the laser by a square wave applied to the $\mathrm{CW}$ laser PZT. ${ }^{15}$ Another possibility is to use a pulse format in which the laser is fired twice during a pumping cycle, giving a pulse separation of several hundred microseconds. ${ }^{16}$ One pulse of this pair can be tuned on line and the other off line. To accomplish rapid wavelength switching in under $100 \mu \mathrm{s}$, we have used an electro-optic switch to select one beam or another generated by two different CW lasers.

\subsection{Receiver}


The pulsed laser output is transmitted to the atmosphere via a 6-inch diameter off-axis paraboloid telescope. After expansion by the telescope, the ouput laser beam can be pointed or scanned by a scanner mounted through the roof of the trailer. The outgoing pulse and atmospheric backscatter are separated by a polarization relationship imposed by the combination of a quarter-wave plate and polarizing beam splitter.

Heterodyne detection is provided by InGaAs photodiodes in a dual-balanced configuration as diagrammed in Figure 4. The dual-balanced design is used here as it allows collection of all of the atmospheric backscatter and local oscillator through a 50/50 evanescent wave coupler. A more typical design would be to use a single photodiode with a coupler taking $90 \%$ of the atmospheric backscatter and $10 \%$ of the local oscillator. The dual-balance configuration has an additional benefit of canceling amplitude noise that may occur in the local oscillator. A photodetector circuit is being used that combines the photodiodes and amplifiers (in die form) integrated together in the same microelectronic package. Such packaging reduces parasitic capacitance, thus improving performance where a wide bandwidth is needed. A factor of 2.2 reduction in parasitic capacitance has been shown over a previous design using discrete components integrated on a printed circuit board. The circuit used in Validar has a $200 \mathrm{MHz}$ bandwidth, but circuits have been developed with $2 \mathrm{GHz}$ bandwidth.

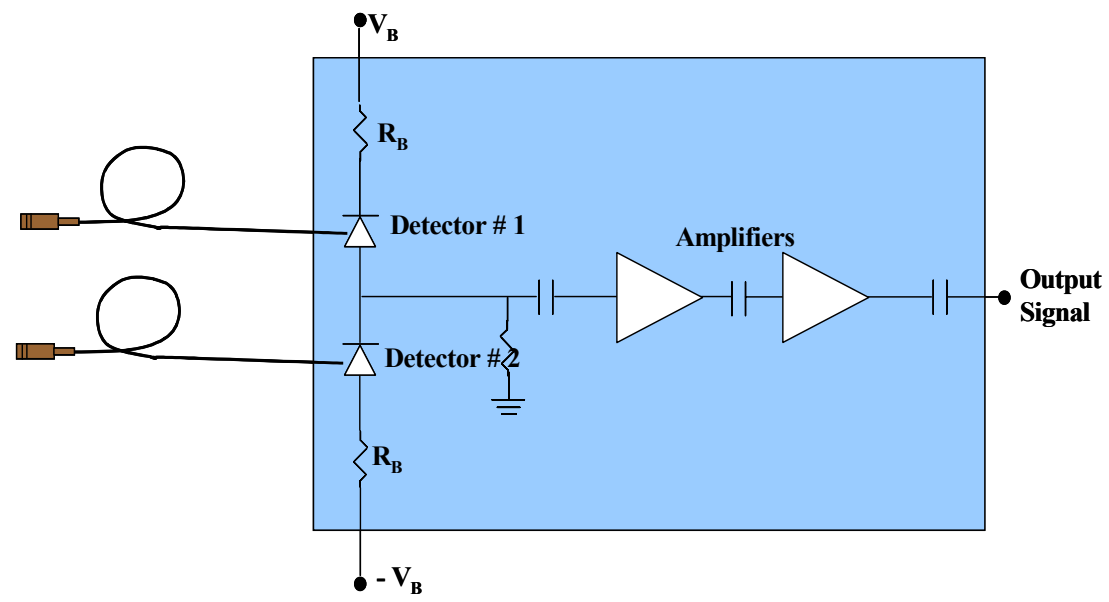

Figure 4: Diagram of integrated photodetector circuit. The components in the shaded region are integrated in a monolithic package. Polarization maintaining fibers deliver light to the InGaAs photodiodes.

\subsection{Data acquisition and signal processing}

Processing of the lidar data is accomplished with the system diagrammed in Figure 5. The heterodyne signal is conditioned by an analog front end that includes an anti-aliasing filter and two different level of amplification switched in to bring the outgoing scatter pulse and atmospheric return within the dynamic range of a digitizer. Sampling by the digitizer is at $500 \mathrm{Ms} / \mathrm{s}$ and 8 bit resolution. An example of a single digitized heterodyne signal is shown in Figure 6 in time domain.

Digitized data flows to an array of SHARC digital signal processors (DSP) for real-time reduction of the heterodyne signal to wind and power data products. The DSP, digitizer, and control software are hosted by a single computer over a PCI backplane. Control software includes a program in which the user can set such parameters as number of sample points, range bin size, number of pulses to be averaged, scanner angles, and display preferences. An outline of the processing steps for an acquisition along a single line of sight is given in the following: 


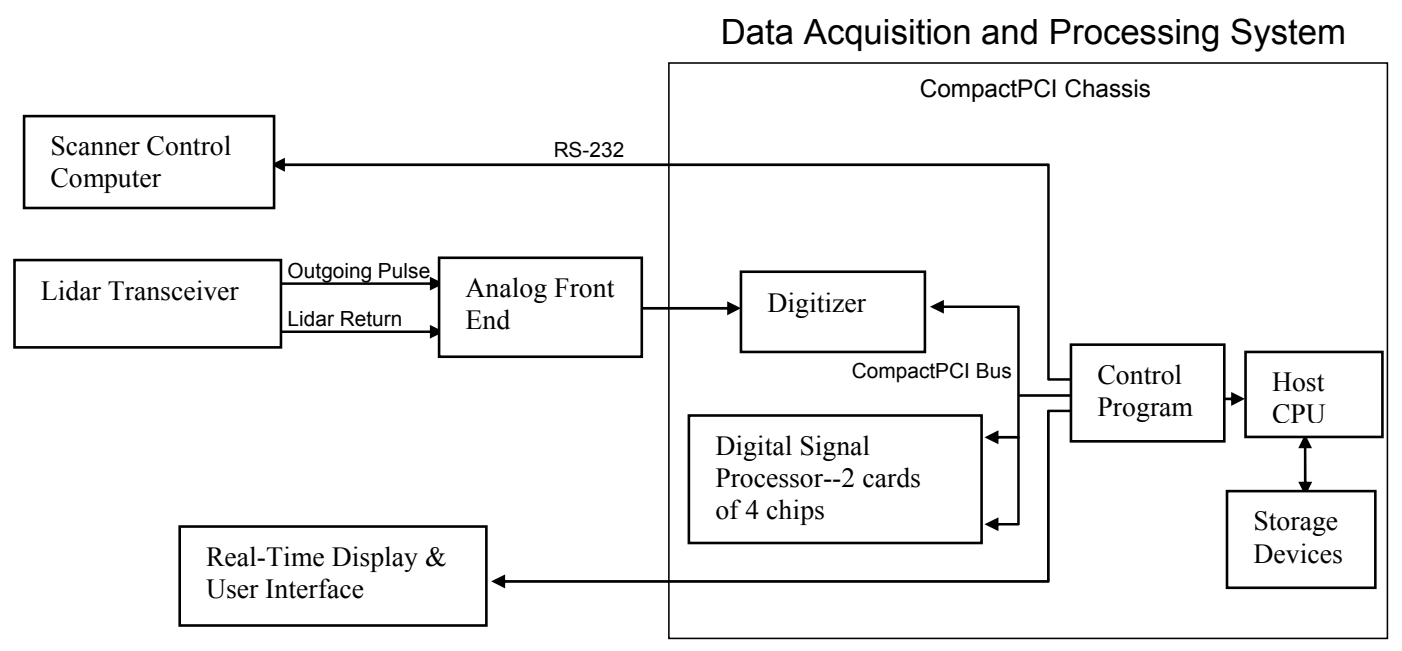

Figure 5: Layout of the Validar electronics and real-time processing system.

\subsubsection{Step (A): acquire}

An individual pulse is digitized starting from a trigger generated by a monitor photodetector. A user-specified number of samples is taken and separated into range bins with an example setup being 50,000 samples broken into 512 sample bins. The first range bin, however, can be of a longer length of 1024 samples to isolate the outgoing pulse scattered from the lidar optics. The scatter pulse is also called a monitor pulse because it is used to monitor for frequency that may occur in the injection seeding process. Range bin information is converted to range in meters. A number of pulses will be acquired for averaging as specified by the user.

\subsubsection{Step (B): FFT}

The power spectrum of the data for each range bin is calculated by a fast Fourier transform (FFT). A Hamming window may be applied as an option.

\subsubsection{Step (C): monitor frequency determination}

The maximum magnitude frequency is estimated in the first range bin containing the monitor pulse. This estimated frequency is compared to a user-specified frequency range to check if the laser pulse was properly injection seeded. An unseeded pulse, which occurs for less than $1 \%$ of the laser pulses, will have a maximum frequency estimate at a frequency corresponding to the free spectral range of the pulsed laser cavity since multiple modes are beating. Such a frequency is well away from the intermediate frequency and is easily filtered by this step. If a bad pulse is detected it is discarded from the set so that it will not be included in averaging.

\subsubsection{Step (D): shift spectra}


Taking the first pulse as the reference, provided it passed the test of the above step, the following pulses are shifted in frequency to remove the effects of pulse-to-pulse frequency jitter. The amount of shifting applied to each pulse is determined from Step $(\mathrm{C})$.

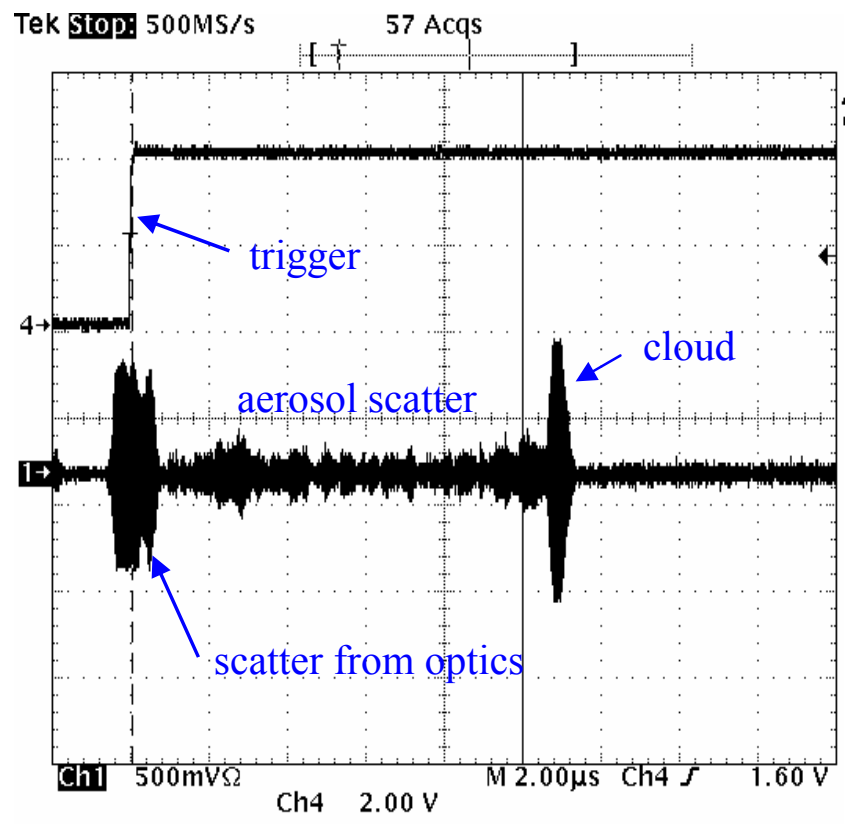

Figure 6: A single pulse return.

\subsubsection{Step (E): averaging}

The jitter-corrected spectra for each range bin are averaged among all the pulses acquired.

\subsubsection{Steo (F): Doppler shit estimation}

The maximum power frequency is determined for each range bin and subtracted from the zero Doppler reference frequency. This result is used to determine the Doppler shift.

\subsubsection{Step (G): power estimation}

The signal power in each range bin is calculated. This information indicates the level of aerosol concentration and the presence of clouds. Power is also used in DIAL measurements in which the atmospheric backscatter is compared at two wavelengths to infer $\mathrm{CO}_{2}$ concentration. Signal processing for coherent DIAL measurements is further described in a report currently in preparation. ${ }^{15}$ 
Each of the above steps may be repeated in different viewing angles. A typical measurement scenario is to steer the laser beam in three directions - two orthogonal azimuths for finding the horizontal wind field and zenith viewing for finding vertical wind motion.

\section{ATMOSPHERIC MEASUREMENTS}

Measurements were made of wind and $\mathrm{CO}_{2}$ during the Spring and Summer of 2003 using a Ho:Tm:YLF laser with 75 $\mathrm{mJ}$ output energy. Upgrades to the lidar, reflected in Table 1, are now being installed and should offer better performance in range capability.

\subsection{Wind measurements}

Vertical wind profiles are shown in Figure 7, with Figure 7a being the case of a cloudy day and Figure 7b being the case of a clear sky. In both these data sets the lidar is pointed toward zenith and wind data is continuously sampled over several minutes with 20 pulses grouped together for averaging and processing. The upper plot in each figure is the wind speed versus altitude and the lower plot is signal power versus altitude. Figure 7 a shows a constant layer of cloud at 8 $\mathrm{km}$ altitude and the passing of occasional cumulus clouds. The wind speed under these cumulus clouds tends to be upward at $1 \mathrm{~m} / \mathrm{s}$, showing the convection of air into the clouds. Aerosol structure can be observed in the signal power with the transition at around $2 \mathrm{~km}$ altitude from the atmospheric boundary layer (ABL) to the free troposphere marked by sharp decrease in signal by over an order of magnitude. As much as two orders of magnitude difference in backscatter between the boundary layer and free troposphere has been observed in other data sets. Velocity measurements in Figure 8a are made consistently up to $6 \mathrm{~km}$ before signal starts to fade into noise. Good measurements pick back up again from clouds starting at around $7.5 \mathrm{~km}$.

The example of Figure $7 \mathrm{~b}$ was taken on a day when no clouds could be observed. However, Validar revealed the presence of sub-visible cirrus at $12.5 \mathrm{~km}$ altitude. Again, a sharp transition in backscatter is seen between the atmospheric boundary layer and free troposphere. Good velocity measurements in this case are made from aerosols up to $5 \mathrm{~km}$ altitude. The vertical wind speed is persistently near zero, as opposed to the day of convective activity of Figure $7 \mathrm{a}$.

A horizontal wind profile can be measured by viewing two orthogonal azimuths at the same elevation, determining speed along each azimuth, and vector summing the components as a function of altitude. In this approach the vertical wind component is assumed to be zero. An example is shown in Figure 8, taken with a 45 degree elevation angle. The wind vector is graphed in two parts as speed and direction. Aerosol backscatter gives good velocity measurements up to $5.5 \mathrm{~km}$. Spurious signals occur at 5.5 to $6 \mathrm{~km}$ from a loss of usable signal-to-noise ratio, such that the frequency estimator returns gibberish. A cloud at $6 \mathrm{~km}$ altitude, though, allows good velocity measurements to be made. Differences can be discerned from this data in the wind within the ABL and above the ABL, with the transition between the two occurring at $2 \mathrm{~km}$ altitude. In the ABL the wind shows a constant speed with changing direction, while the free troposphere shows an increasing speed with a constant direction. Increasing speed with altitude is to be expected as friction with the ground has less influence.

The data acquisition and control software, described in Section 2, can automate the collection of data similar to Figures 7 and 8. A typical measurement scenario is to view three different angles with 20 pulses averaged at each angle. At 5 $\mathrm{Hz}$ pulse repetition rate such a measurement set requires 15-20 seconds to complete depending on the extent of data archiving selected. This measurement set, which provides horizontal and vertical wind profiles, can then be automatically repeated. 


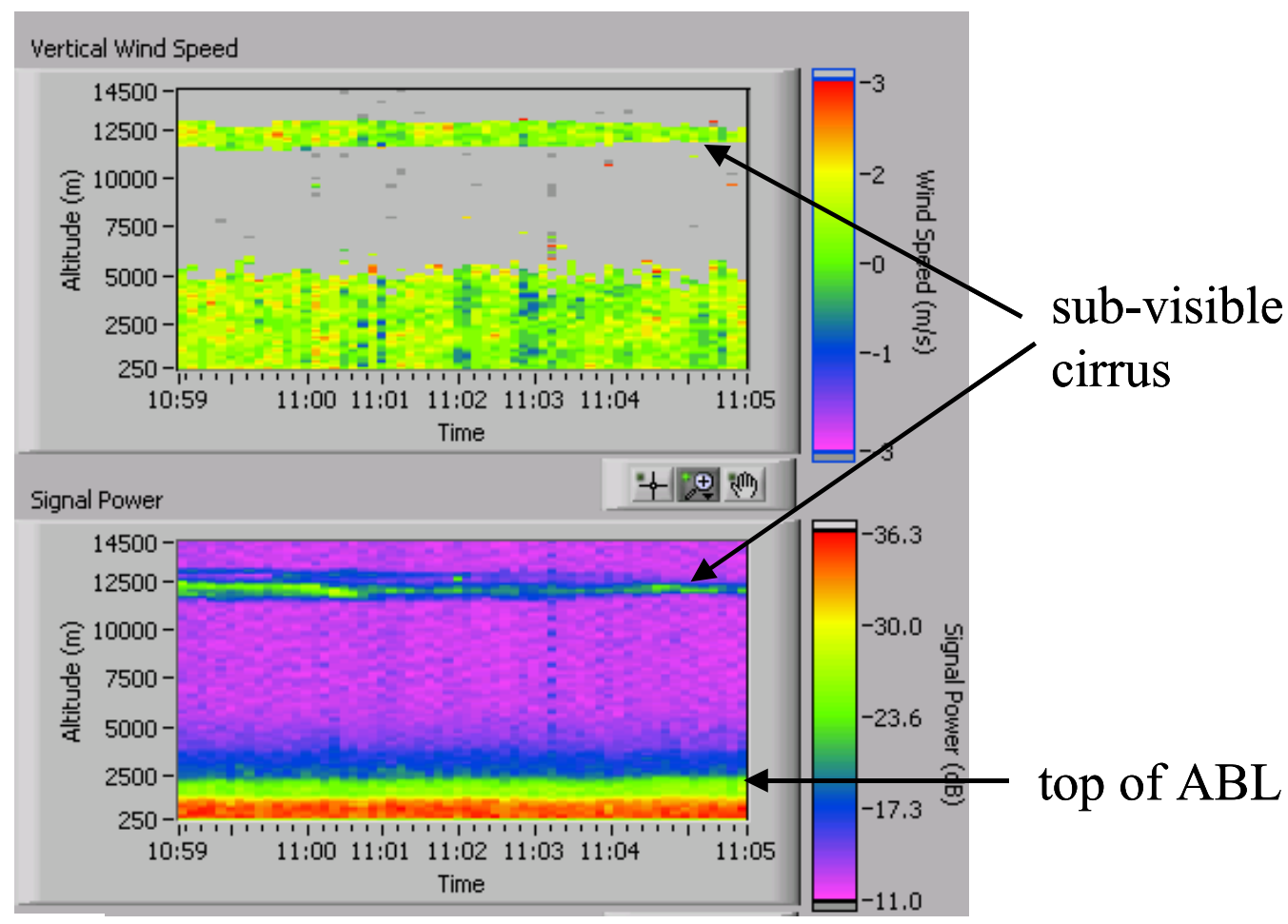

(a)

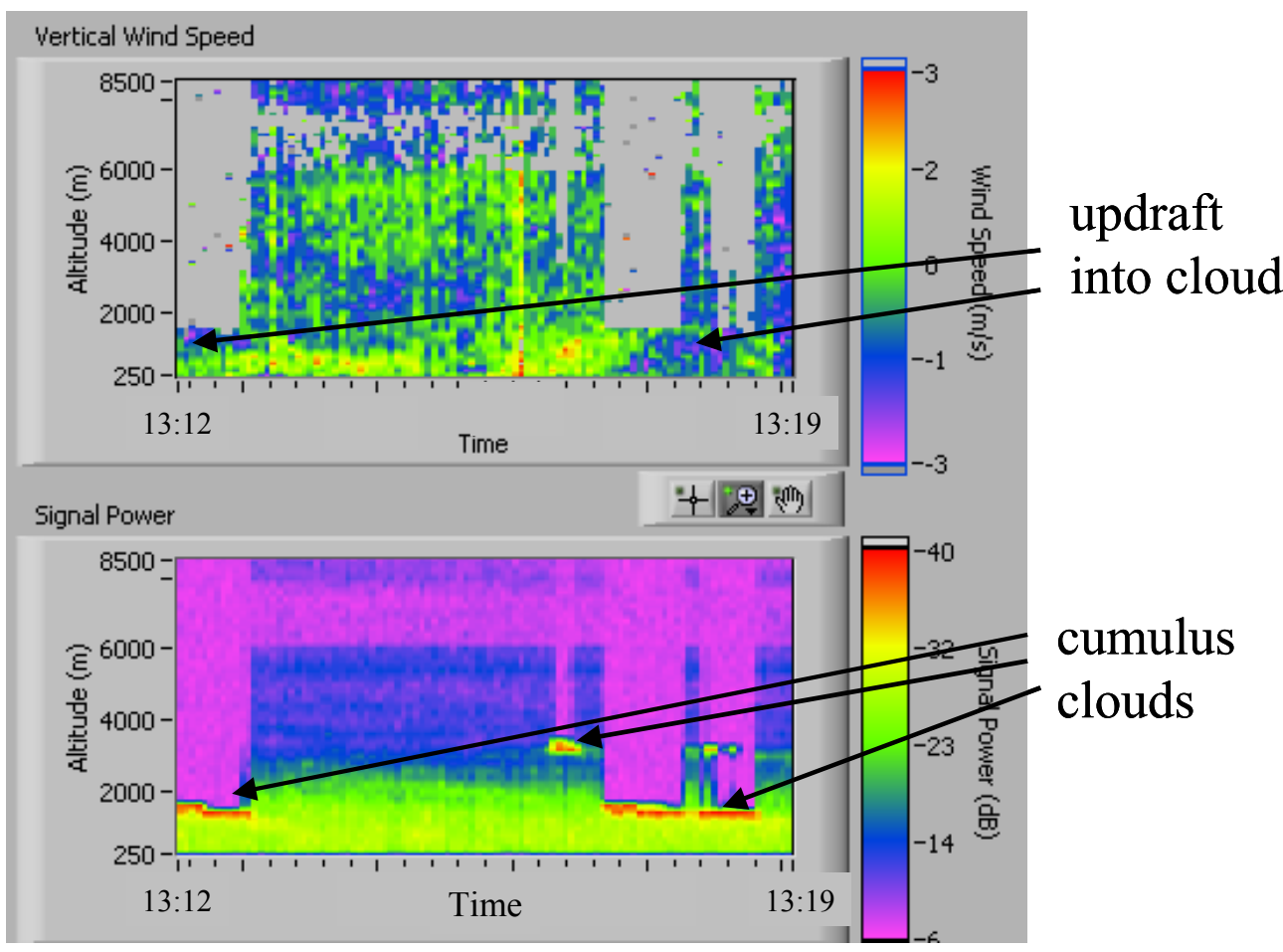

Figure 7: Vertical wind speed time history for (a) cloudy conditions and (b) clear sky. Velocity is shown in the upper graph of each data set. Power of the atmospheric return is the lower graph. 


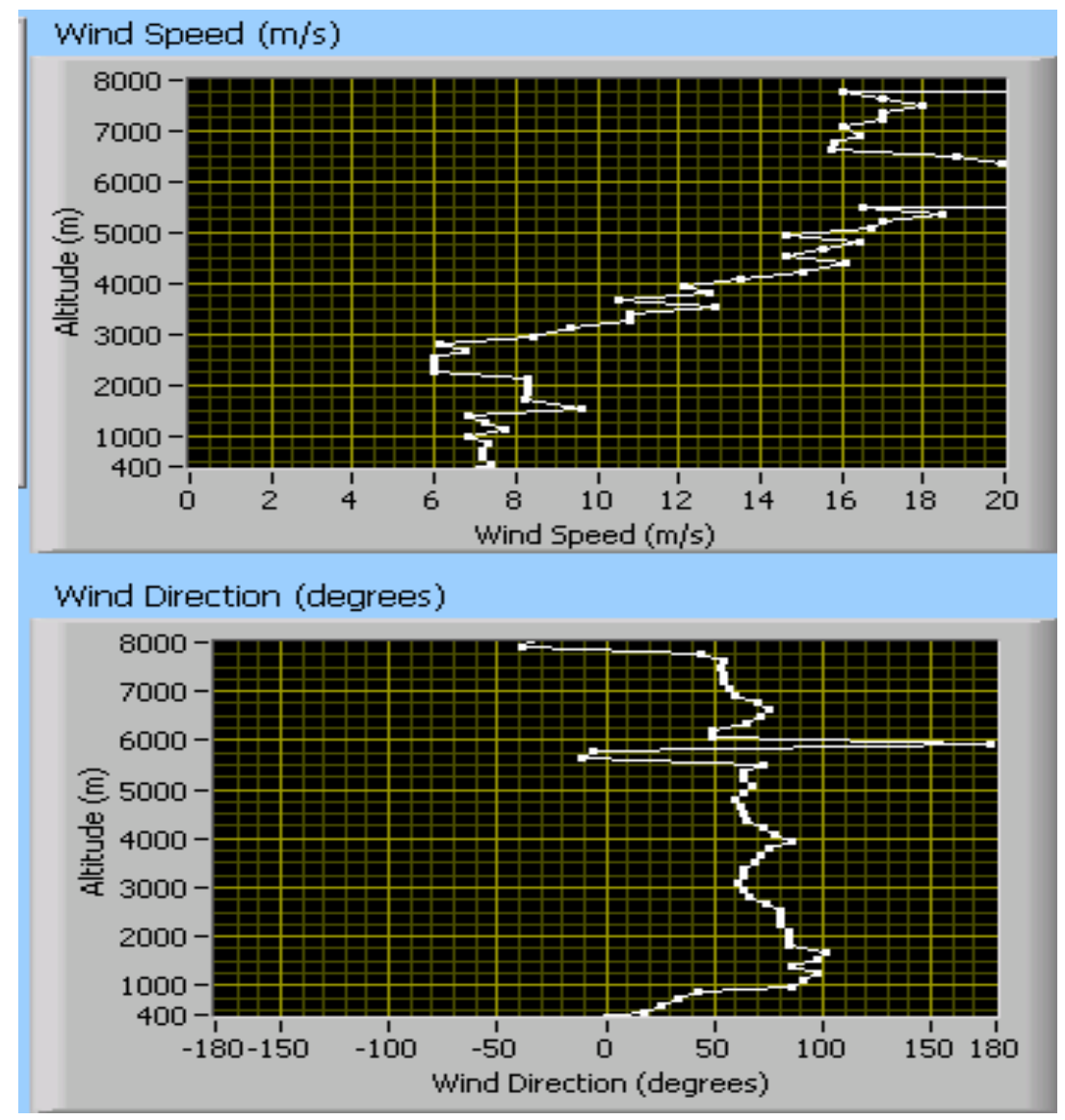

Figure 8: Horizontal wind profile.

\section{2. $\mathrm{CO}_{2}$ measurements}

The configuration of Validar for $\mathrm{CO}_{2}$ measurement is similar to that for wind measurements, with the addition of precise wavelength control and alternation among laser pulses between wavelengths on and off an absorption peak. Signal processing aspects are also different for DIAL in that backscatter power measurements are required rather than spectral shift information used for wind measurement. A detailed report of recent implementation of these technologies is in preparation, and only a brief overview is given here. ${ }^{15}$

One challenge in precise power measurement from a heterodyne signal is that the power return from a particular range depends on the phase of the atmospheric backscatter relative to the local oscillator. This effect, called speckle, creates a power fluctuation from pulse to pulse. Averaging of many pulses is required to reduce the effect of speckle -1000 
pulses were averaged in our experiment at each of two wavelengths. Since the two wavelengths are alternated, a single DIAL measurement requires 2000 consecutive pulses. Processing of the DIAL data separates the 2000 pulses into pairs with, for example, the odd numbered pulses being the shots taken with wavelength on line and the even numbered pulses off line. Strict consecutive acquisition must be observed over these 2000 pulses, without skipping a pulse that would ruin the alternated wavelength sequence.

Processing of the DIAL data uses the same steps for wind data described in Section 2, with the power estimation from each range bin being the information of interest. The best approach found for power measurement involves integrating frequency domain spectra over a threshold set just above the noise floor. Figure 9 is an example of this power measurement. The on-line case falls off at a faster rate with range than the off-line case as a result of strong atmospheric absorption on line. Comparison of the rate of attenuation of the on-line data and off-line data gives a measure of $\mathrm{CO}_{2}$ concentration. Extensive tests were run to assess the precision in measuring $\mathrm{CO}_{2}$ and to show that a diurnal variation of $\mathrm{CO}_{2}$ could be detected. ${ }^{15}$

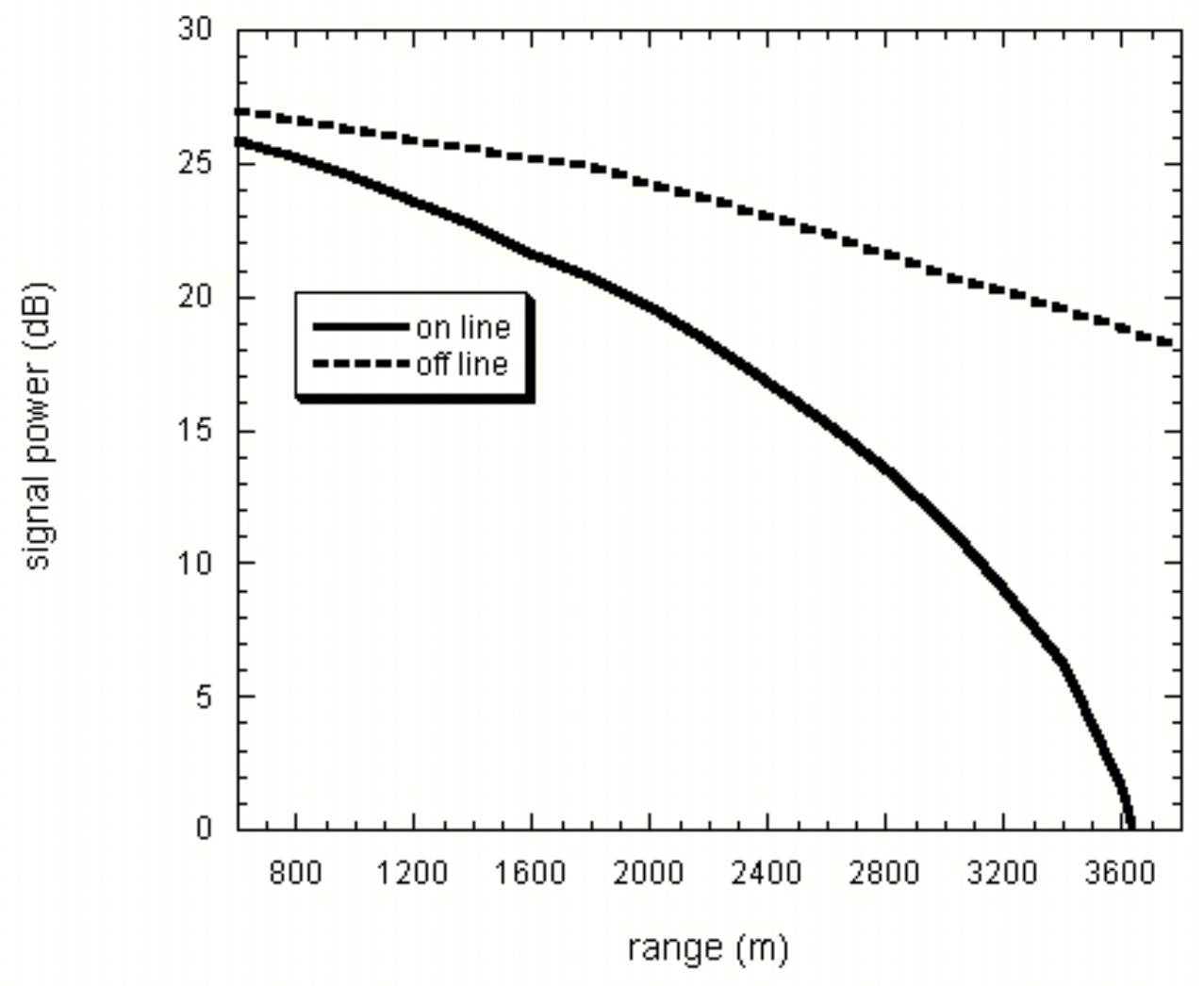

Figure 9: Power in the atmospheric return for on-line and off-line wavelengths. 1000 pulses were averaged for each wavelength. 


\section{ACKNOWLEGEMENTS}

This work was supported by the NASA Laser Risk Reduction Program and the Integrated Program Office.

\section{REFERENCES}

1. S.M. Hannon, S.W. Henderson, J.A. Thomson, and P. Gatt, "Autonomous lidar wind field sensor: performance predictions," SPIE Volume 2832, pp. 76-91 (1996).

2. C.J. Grund, R.M. Banta, J.L. George, J.N. Howell, M.J. Post, R.A. Richter, A.M. Weickmann, "HighResolution Doppler Lidar for Boundary Layer and Cloud Research," J. Atmos. And Ocean. Tech, 18, 376-393 (2001).

3. P. Brockman, B.C. Barker, G.J. Koch, D.P.C. Nguyen, and C.L. Britt, "Coherent pulsed lidar sensing of wake vortex position and strength, winds and turbulence in airport terminal areas," Tenth Biennial Coherent Laser Radar Technologies and Applications Conference, pp. 12-15 (1999).

4. W. Baker, G.D. Emmitt, F. Robertson, R. Atlas, J. Molinari, D. Bowdle, J. Paergle, R.M. Hardesty, R. Menzies, T. Krishnamurti, R. Brown, M.J. Post, J. Anderson, A. Lorenc, and J. McElroy, "Lidar-Measured Winds from Space: A Key Component for Weather and Climate Prediction," Bull. Amer. Meteor. Soc. 76, 869-888 (1995).

5. R.J. Fleming, "National environmental observing system to mitigate the effects of a nuclear-biologicalchemical (NBC) attacks: strategic and tactical," SPIE Volume 5071, pp. 22-32 (2003).

6. C.D. Keeling, "Atmospheric carbon dioxide variations at Mauna Loa Observatory, Hawaii," Tellus 25, 538551 (1976).

7. J. Kaiser and K. Schmidt, "Coming to grips with the world's greenhouse gases," Science 281, 504-506 (1999).

8. American National Standard Z136.1-1993.

9. U.N. Singh, J. Yu, M. Petros, N.P. Barnes, J.A. Williams-Byrd, G.E. Lockard, and E.A. Modlin, "Injectionseeded, room-temperature, diode-pumped Ho:Tm:YLF laser with output energy of $600 \mathrm{~mJ}$ at $10 \mathrm{~Hz}$," in Advanced Solid-State Lasers, Vol. 19 of OSA Trends in Optics and Photonics, pp. 194-196 (1998).

10. M. Petros, J. Yu, S. Chen, U.N. Singh, B.M. Walsh, Y. Bai, and N.P. Barnes, "High energy diode pumped Ho:Tm:LuLiF 4 laser for lidar applications," SPIE Vol. 4893, pp. 203-210 (2002).

11. M.J. Kavaya and G.D Emmitt, "The Space Readiness Coherent Lidar Experiment (SPARCLE)," paper 338003, Proc. SPIE Vol. 3380, pp. 2-11 (1998).

12. S.W. Henderson, E.Y. Yuen, and E.S. Fry, "Fast resonance detection technique for single-frequency operation of injection seeded Nd:YAG lasers," Opt. Lett. 11, 715-717 (1986).

13. G.J. Koch, A.N. Dharamsi, C.M. Fitzgerald, and J.C. McCarthy, "Frequency stabilization of a Ho:Tm:YLF laser to absorption lines of carbon dioxide," Appl. Opt. 39, 3664-3669 (2000).

14. G.J. Koch, M. Petros, J.Yu, and U.N. Singh, "Precise wavelength control of a pulsed single-frequency Ho:Tm:YLF laser," Appl. Opt. 41, 1718-1721 (2002).

15. G.J. Koch, B.W. Barnes, M. Petros, J.Y. Beyon, F. Amzajerdian, J. Yu, R.E. Davis, S. Ismail, S. Vay, M.J. Kavaya, and U.N. Singh, "Coherent Differential Absorption Lidar Measurement of $\mathrm{CO}_{2}$," Appl. Opt. under review (2004).

16. J. Yu, U.N. Singh, J.C. Barnes, N.P. Barnes, and M. Petros, "An Efficient Double Pulsed 2-micron Laser for DIAL Applications," in Advances in Laser Remote Sensing: Selected Papers presented at the $20^{\text {th }}$ International Laser Radar Confernce (ILRC) Vichy, France, 10-14 July 2000, Editors A. Dabas, C. Loth, and J. Pelon, ISBN 2-7302-0798-8 Editions de l'Ecole polytechnique. 